\title{
V453 Oph: a s-process enriched, but carbon-deficient RV Tauri star of low intrinsic metallicity $\star$
}

\author{
P. Deroo ${ }^{1}$, M. Reyniers ${ }^{1}$, H. Van Winckel ${ }^{1}$, S. Goriely ${ }^{2}$, and L. Siess ${ }^{2}$ \\ 1 Instituut voor Sterrenkunde, KU Leuven, Celestijnenlaan 200B, 3001 Leuven, Belgium \\ e-mail: Pieter.Deroo@ster.kuleuven.be \\ 2 Institut d'Astronomie et d'Astrophysique, Université Libre de Bruxelles, CP 226, 1050 Brussels, Belgium
}

Received 18 August 2004 / Accepted 1 April 2005

\begin{abstract}
This paper reports the detection of a heavy element enriched RV Tauri variable with an abundance pattern that differs significantly from a standard s-process enriched object: V453 Oph. Based on optical high-resolution spectra, we determined that this object of low intrinsic metallicity $([\mathrm{Fe} / \mathrm{H}]=-2.2)$ has a mild, but significant, enrichment $([\mathrm{s} / \mathrm{Fe}] \sim+0.5)$ of heavy elements for which the distribution points to slow neutron capture nucleosynthesis. This result is strengthened by a comparative analysis to the non-enriched RV Tauri star DS Aqr ([s/Fe] = 0.0). Although V453 Oph is the first RV Tauri star showing a strong s-process signature, it is not accompanied by $\mathrm{C}$ enhancement, challenging our current nucleosynthetic models of postAGB stars that predict a simultaneous enrichment in $\mathrm{C}$ and s-process elements. The low $\mathrm{N}$ abundance excludes $\mathrm{CN}$ cycling as being responsible for the low $\mathrm{C}$ abundance. We explore three different scenarios to explain the heavy element distribution in this evolved object: an enrichment of the parental cloud, an accretion scenario in which the chemical patterns were acquired by mass transfer in a binary system and an intrinsic enrichment by dredge-up.
\end{abstract}

Key words. stars: abundances - stars: evolution - stars: AGB and post-AGB - nuclear reactions, nucleosynthesis, abundances - stars: individual: V453 Oph - stars: individual: DS Aqr

\section{Introduction}

RV Tauri stars are rare supergiants that occupy the highluminosity end of the pop. II Cepheid instability strip. The identification of RV Tauri stars is based on their characteristic light curves, which show alternating deep and shallow minima with periods ranging from 30 to 150 days. This variability is often accompanied with lower amplitude irregularities and cycle-to-cycle variability (e.g. review on pop. II Cepheids by Wallerstein 2002).

The far-IR excess of RV Tauri stars due to circumstellar dust was detected by IRAS and led Jura (1986) to conclude that they are young post-AGB stars, in which the circumstellar dust is a relic of the strong dusty mass loss on the AGB. For many RV Tauri variables, a near-IR excess is detected as well (Gehrz 1972; Evans 1985). The genuine high-luminosity nature of the RV Tauri class was confirmed by the detection of some members in Globular Clusters and in the LMC. The LMC variables obey a period-luminosity $(\mathrm{P}-\mathrm{L})$ relation, which follows the high luminosity extension of the $\mathrm{P}-\mathrm{L}$ relation of W Vir stars (Alcock et al. 1998).

* Based on observations collected at the European Southern Observatory (La Silla) in Chile (67.D-0054(A) and on the $1.2 \mathrm{~m}$ Swiss Euler telescope also on La Silla.
Chemically, however, RV Tauri stars do not show evidence for post-third dredge-up enrichment: no high C-abundances either s-process overabundances due to a third dredge-up have been found. A summary of the available quantitative abundance data on RV Tauri stars can be found in Giridhar et al. (2000) and Maas et al. (2005). Many RV Tauri stars show evidence of selective depletion in which the refractory elements are depleted relative to the non-refractory ones. These photospheric chemical patterns are the result of a poorly understood process in which the circumstellar dust is separated from the circumstellar gas, followed by a selective re-accretion of the non-refractories (Mathis \& Lamers 1992; Waters et al. 1992). Important diagnostics for this process are high $[\mathrm{Zn} / \mathrm{Fe}],[\mathrm{Zn} / \mathrm{Ti}]$ and $[\mathrm{S} / \alpha]$ ratios, because $\mathrm{Zn}$ and $\mathrm{S}$ have a very low condensation temperature with respect to $\mathrm{Fe}, \mathrm{Ti}$ and the $\alpha$-elements . The process will also result in a $\mathrm{C} / \mathrm{O}$ ratio larger than solar due to the stability of the $\mathrm{CO}$ molecule, which is thought to be abundant in the gas accreted onto the stellar surface. Since s-process elements are refractory, a subsequent depletion process can mask an intrinsic AGB enrichment. Nevertheless, overabundances of s-process elements relative to other species with similar condensation temperature were undetected.

The depletion process can be very efficient in post-AGB binary stars for which there is evidence that the dust is trapped in a circumstellar disc (Van Winckel et al. 1995). This 
Table 1. Some basic parameters of V453 Oph and DS Aqr. The pulsation periods, spectral types and magnitude ranges are from Kholopov et al. (1998). The other parameters were obtained from Simbad.

\begin{tabular}{lrrr}
\hline \hline \multicolumn{1}{c}{ Data } & & \multicolumn{1}{c}{ V453 Oph } & \multicolumn{1}{c}{ DS Aqr } \\
\hline Other name: & & BD-02 4345 & HD 216457 \\
Coordinates: & $\alpha_{2000}$ & +172649.13 & +225317.04 \\
& $\delta_{2000}$ & -022336.44 & -183530.99 \\
Min. mag.: & & $10.4 \mathrm{mag}$ & $11.08 \mathrm{mag}$ \\
Max. mag.: & & $11.53 \mathrm{mag}$ & $11.90 \mathrm{mag}$ \\
Spectral type: & & Fp & F2II \\
Period: & & $81.3 \mathrm{~d}$ & $78.213 \mathrm{~d}$ \\
Galactic & $l$ & 20.72 & 44.10 \\
Coordinates: & $b$ & 17.57 & -61.56 \\
\hline
\end{tabular}

geometry may well be a necessary condition for the depletion process to be efficient (Waters et al. 1992). Evidence is growing that also in dusty RV Tauri stars the dust is trapped in a stable disc (Evans 1985; Van Winckel et al. 1999a) and the binary nature of several dusty RV Tauri stars has been established (Van Winckel 2003, and references therein). It is, however, not generally accepted that all dusty RV Tauri stars reside in binaries. There is no clear relation between the current IR-excess and the strength of the depletion process (Giridhar et al. 2000).

The traditional spectroscopic classification of RV Tauri stars by Preston et al. (1963) is strongly determined by the efficiency of the depletion process. Spectroscopic group A consists of stars of spectral type G or K, showing strong absorption lines but normal $\mathrm{CN}$ or $\mathrm{CH}$ bands, while some also show $\mathrm{TiO}$ bands at minimum light. Group B stars, being generally somewhat hotter, show weak metal lines combined with enhanced $\mathrm{CN}$ and $\mathrm{CH}$ bands. Members of group $\mathrm{C}$ resemble those of group $\mathrm{B}$, except that enhanced bands of $\mathrm{CN}$ are not seen. It is now clear that the low metallicity of the weak-lined group B stars is determined by the efficiency of the depletion process. Depletion patterns are less strong, if present at all, in group A stars. Group $\mathrm{C}$ stars are not affected by depletion, probably due to their low intrinsic metallicity (Giridhar et al. 2000).

In this paper we focus on the chemical analysis of two RV Tauri stars, V453 Oph and DS Aqr of spectroscopic class $\mathrm{C}$ to study potential AGB enrichment. The luminosity of these stars is about $2400 \pm 1600 L_{\odot}$, as determined using the $\mathrm{P}-\mathrm{L}$ relation of RV Tauri variables in the LMC (Alcock et al. 1998). This high luminosity identifies them as likely post-AGB stars (e.g. Bloecker 1995). Since class C objects are not affected by the depletion process, these intrinsically metal-poor objects are ideal to study AGB enrichment and possibly the metallicity dependence of the s-process. Some basic data of both stars are shown in Table 1.

After an overview of the observations, we discuss our spectroscopic parameter determination in Sect. 3. The abundance pattern for V453 Oph and DS Aqr is derived in Sect. 4 and discussed in Sect. 5. In Sects. 6 and 7 we explore different scenarios to explain the observed abundances. We end with the conclusions in Sect. 8.
Table 2. The $\log$ of the obtained FEROS spectra together with the signal-to-noise $(S / N)$ ratio in different spectral regions. The $S / N$ ratio listed for V453 Oph corresponds to the final spectrum.

\begin{tabular}{clccc}
\hline \hline Name & Date and UT & $\begin{array}{c}S / N \\
400 \mathrm{~nm}\end{array}$ & $\begin{array}{c}S / N \\
585 \mathrm{~nm}\end{array}$ & $\begin{array}{c}S / N \\
770 \mathrm{~nm}\end{array}$ \\
\hline V453 Oph & $\begin{array}{l}28 / 06 / 0105: 30 \\
28 / 06 / 0106: 25\end{array}$ & 50 & 180 & 180 \\
DS Aqr & $27 / 06 / 0109: 05$ & 50 & 110 & 80 \\
\hline
\end{tabular}

\section{Observations}

\subsection{Spectroscopic}

High-resolution spectra of both RV Tauri variables were acquired with the fibre fed spectrograph FEROS, mounted on the $1.5 \mathrm{~m}$ ESO telescope at La Silla. The resolving power of the spectra is $R \sim 48000$ at $5000 \AA$ and a wavelength coverage ranging from $3600 \AA$ to $9200 \AA$ is obtained in a single exposure. Two exposures were obtained for V453 Oph and one for DS Aqr. The log of the observations is reported in Table 2.

The reduction of the spectra was performed in the standard fashion, using the FEROS DRS package in the MIDAS environment. Our reduction includes bias correction, background subtraction, cosmic hit cleaning, flatfielding, order extraction and wavelength calibration. Ultimately, the spectra of the different orders were merged and normalised.

For V453 Oph, the two normalised spectra were averaged, weighted by their signal-to-noise $(S / N)$ ratio. In Table 2 the $S / N$ ratio is given for different spectral regions. Sample spectra are shown in Figs. 5-7.

Additionally, we monitored DS Aqr with the CORALIE spectrograph mounted on the Swiss telescope at La Silla, Chile. CORALIE (Queloz et al. 1999) is a high resolution $(R \sim 50000)$ fibre-fed echelle spectrograph specifically constructed to obtain high precision radial velocities through a cross-correlation technique. We started the monitoring in August 2000 and obtained 40 data points.

\subsection{Photometric}

Using the light curve ephemeredes of Kholopov et al. (1998), we estimate that the spectra of V453 Oph and DS Aqr were taken at pulsation phase $\phi=0.4$ and $\phi=0.0$ respectively. Unfortunately, no simultaneous photometric data over a large wavelength range could be obtained for these phases. Instead we used photometric data obtained around the phases $\phi=0.55$ and $\phi=0.3$ respectively. These data are reported in Table 3 .

The total reddening was determined minimising the difference between model atmospheres and dereddened fluxes in the optical wavelength range. For the model atmospheres, Kurucz models with parameters determined in Sect. 3 were used. This is possible because small photometric phase discrepancies are only expected to result in small shifts in atmospheric parameters.

For V453 Oph, we obtain a total reddening of $E(B-V)=$ 0.50 and for DS Aqr, $E(B-V)=0.05$. The resulting SED for 
Table 3. The photometric measurements of V453 Oph and DS Aqr. The data are taken from various authors and plotted in Fig. 1: ${ }^{a}$ data from Goldsmith et al. (1987); ${ }^{b}$ data from the 2MASS catalogue; ${ }^{c}$ data from Gehrz (1972).

\begin{tabular}{lrrrr}
\hline \hline Band & V453 Oph (mag) & \multicolumn{2}{c}{ DS Aqr (mag) } \\
\hline$U$ & $11.76^{a}$ & $11.66^{a}$ & \\
$B$ & $11.33^{a}$ & $11.18^{a}$ & \\
$V$ & $10.54^{a}$ & $10.51^{a}$ & \\
$R$ & $9.99^{a}$ & & $10.12^{a}$ & \\
$I$ & $9.39^{a}$ & & $9.73^{a}$ & \\
$\mathrm{~J}$ & $8.58^{a}$ & $8.806^{b}$ & $9.16^{a}$ & $9.488^{b}$ \\
$2.2 \mu \mathrm{m}$ & & $7.6^{c}$ & & \\
$H$ & $8.09^{a}$ & $8.442^{b}$ & $8.79^{a}$ & $9.197^{b}$ \\
$K$ & $7.95^{a}$ & $8.283^{b}$ & $8.72^{a}$ & $9.089^{b}$ \\
$L$ & $>7.0^{a}$ & & $>7.0^{a}$ & \\
$M$ & $>6.3^{a}$ & & $>6.3^{a}$ & \\
$N$ & $>4.5^{a}$ & & $>4.5^{a}$ & \\
$11.3 \mu \mathrm{m}$ & \multicolumn{4}{c}{$>4.6^{c}$} \\
\hline
\end{tabular}

both stars is shown in Fig. 1. No near-infrared excess was detected for either variable. The high reddening of V453 Oph is consistent with the $E(B-V)$ obtained from the diffuse interstellar bands at $\lambda=5780 \AA$ and $5797 \AA$, of which the equivalent width correlates well with this parameter (Josafatsson \& Snow 1987). Using the data reported in Krełowski et al. (1999) these bands give an $E(B-V)=0.48$ and 0.54 respectively for V453 Oph.

\section{Parameter determination}

\subsection{Atomic data}

For the abundance analysis, all lines showing a clear symmetric profile were measured. These lines were identified, based on wavelength, using mainly the solar line list of Thévenin (1989). This list was also employed to identify blended lines using the calculated equivalent width (EW) of all lines in the spectral region. To calculate the abundances of the different elements, atomic data were preferably taken from the list with accurate oscillator strengths for $\mathrm{A}$ to $\mathrm{F}$ type stars, which has been collected at the Instituut voor Sterrenkunde (Van Winckel \& Reyniers 2000; Reyniers et al. 2004). Alternatively, to obtain accurate oscillator strengths, the line lists of the Vienna Atomic Line Database (VALD2) were used (http://www.astro.univie.ac.at/ vald/, Kupka et al. (1999)). Additionally, the line list of the DREAM project (http://www. umh.ac.be/ astro/dream.html) was used for $\mathrm{Ce}$, while for $\mathrm{La}, \mathrm{Eu}$ and Dy, data reported in respectively Lawler et al. (2001a), Lawler et al. (2001b) and Biémont \& Lowe (1993) were used.

\subsection{Radial velocities}

The heliocentric radial velocities for V453 Oph and DS Aqr were determined using Fe lines as $-123.8 \pm 0.6 \mathrm{~km} \mathrm{~s}^{-1}$ and $-23.9 \pm 0.8 \mathrm{~km} \mathrm{~s}^{-1}$ respectively. These values differ respectively only by $5 \mathrm{~km} \mathrm{~s}^{-1}$ and $3 \mathrm{~km} \mathrm{~s}^{-1}$ from published values (Giridhar et al. 1998).
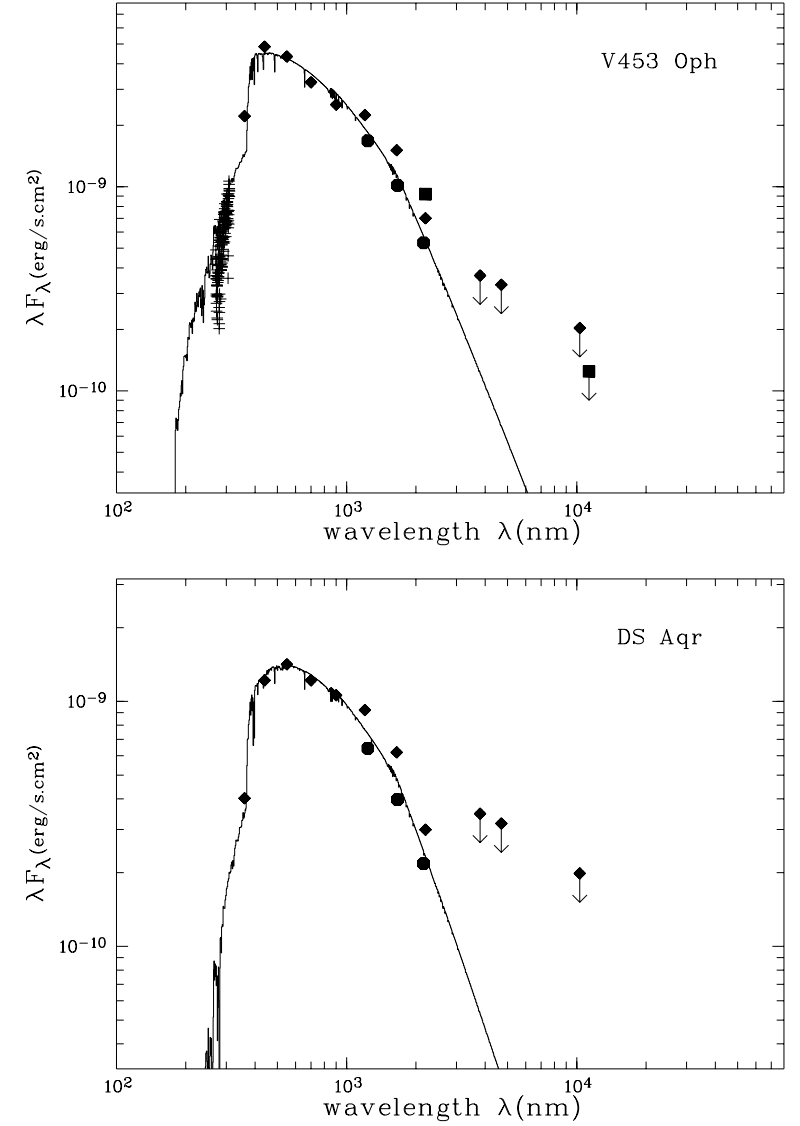

Fig. 1. The SED for V453 Oph (top) and DS Aqr (bottom), constructed using a total reddening of $E(B-V)=0.50,0.05$ respectively. The full line is the Kurucz model, while the other symbols are photometric measurements from various authors, listed in Table 3. Upper limits are marked with arrows down. The plus signs are taken from the IUE archive, the diamonds from Goldsmith et al. (1987), the circles from the 2MASS archive and the squares are from Gehrz (1972). The figure shows clearly that no near-IR excess is observed.

For DS Aqr, we have also 40 high resolution, low signalto-noise CORALIE spectra (see Sect. 2.1). In the online reduction of these spectra, radial velocities are determined by cross-correlation with a standard F0 mask. Since many of the correlation profiles were quite noisy, we decided to construct a mask optimized for DS Aqr. This technique is proven to be very efficient for other RV Tauri stars (Maas 2003). The special mask contains only the lines that were used in the abundance study (Sect. 4). In Fig. 2 we compare the correlation profiles for two spectra obtained by using the standard mask and by using our constructed mask. Their smoother profile and their deeper central depth clearly indicate the higher quality of the latter. Six out of the 40 spectra were of a too low signal-to-noise to obtain a reliable velocity.

The remaining 34 velocities for DS Aqr display a large peak-to-peak amplitude of $26 \mathrm{~km} \mathrm{~s}^{-1}$ (see Fig. 3) Such a high value is not uncommon even for single RV Tau stars, and does not necessarily suggest a binary nature of the object. The photometric phase distribution of our 34 velocities is poor and since cycle-to-cycle variability is often observed in RV Tauri stars, 

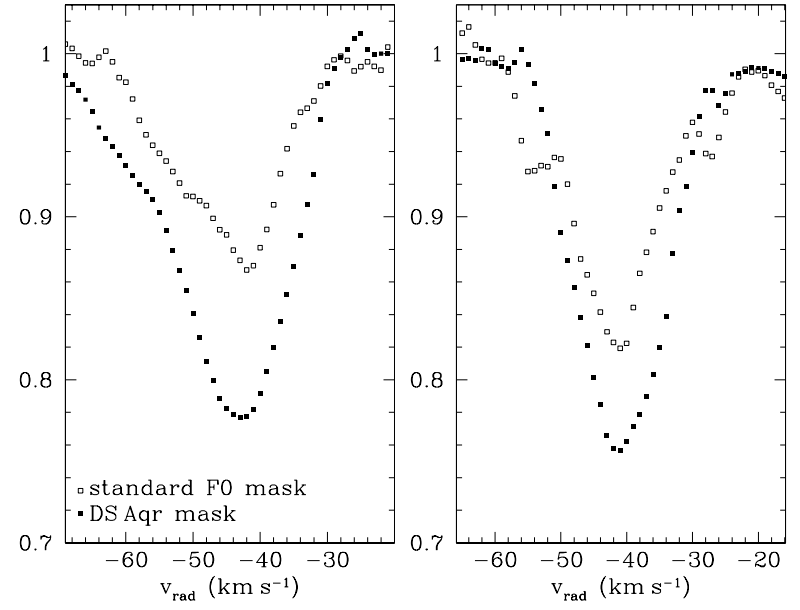

Fig. 2. Cross-correlation profiles for DS Aqr. Comparison of the crosscorrelation profiles for two CORALIE spectra, obtained with a standard mask (open squares) and a specific template for DS Aqr (full squares). The profiles on the left panel are for the spectrum taken on $21 / 07 / 2001$, the ones on the right panel for the spectrum of $25 / 07 / 2001$

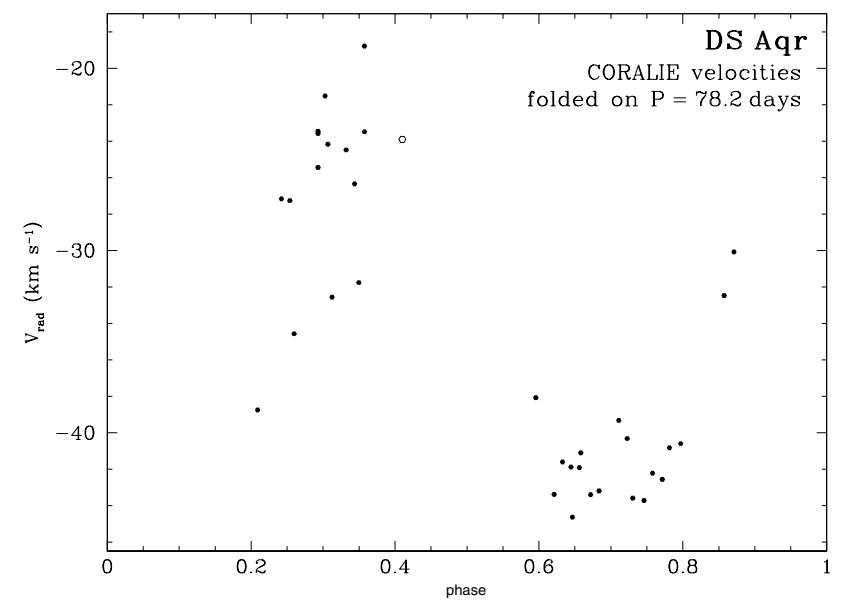

Fig. 3. Phase diagram of the 34 CORALIE velocities (filled circles) and the velocity obtained from the FEROS spectrum (open circle) of DS Aqr. The velocities are folded on the photometric period of 78.213 days that was published by Kholopov et al. (1998). Since cycle-to-cycle intrinsic variability is often observed in RV Tauri stars, a much better sampling is needed to be able to test for binary motion.

we have as yet no significant test to probe the eventual binary nature of DS Aqr.

Concerning the radial velocities of the two programme stars, we can conclude that the variability of the radial velocities can be accounted for by the known photometric variability of the stars, with peak-to-peak amplitudes in $\mathrm{V}$ of 1.1 mag for V453 Oph and $0.8 \mathrm{mag}$ for DS Aqr.

\subsection{Determination of atmospheric parameters}

The abundance pattern was computed using the latest ATLAS9 LTE model atmospheres (Castelli \& Kurucz 2004) in combination with the LTE line analysis program MOOG of Sneden (version 2002). The model atmospheres are uniquely

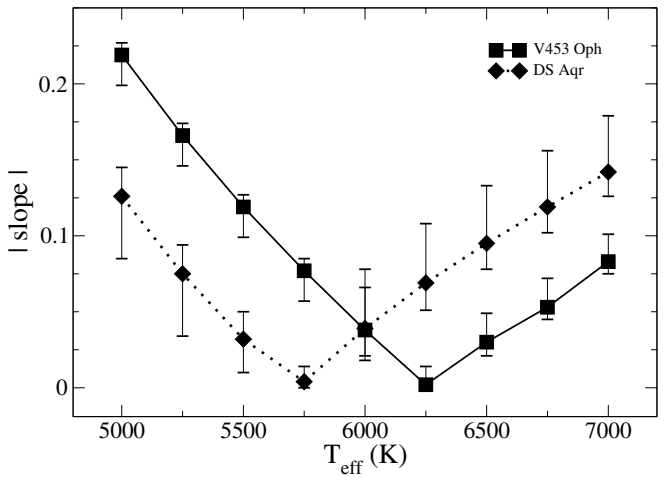

Fig. 4. The slope of the trend between the abundance derived from $\mathrm{Fe}$ I lines and the EP versus temperature. The values for V453 Oph are depicted using squares and those for DS Aqr using diamonds. These values were determined using $\log (g)=1.5$, and $\log (g)=0.5$ respectively. An estimate on the errors was made by varying the microturbulent velocity by $\pm 1 \mathrm{~km} \mathrm{~s}^{-1}$.

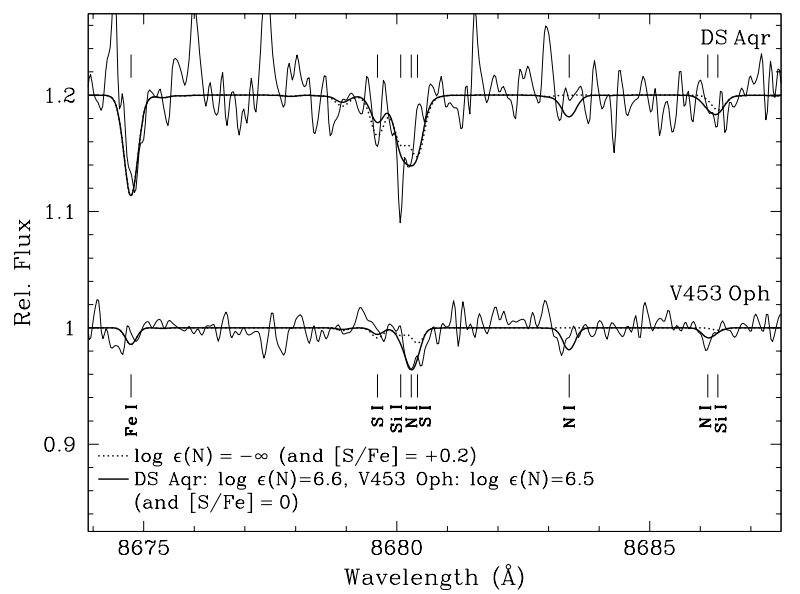

Fig. 5. Derivation of the upper limit for the nitrogen abundance, using spectrum synthesis of the N I triplet at $8680 \AA$. Two syntheses for each star were made: one without any nitrogen, to detect possible blends (dotted line), and the other one with the proposed upper limit abundance for nitrogen (thick continuous line). From the first synthesis, it turned out that the strongest NI line at $8680.28 \AA$ is not reliable to deduce an $\mathrm{N}$ abundance, due to weak Si I and S I blends. Therefore, the upper limits obtained in the second synthesis focus on the weaker line at $8683.40 \AA$, being $\log \epsilon(N)<6.6$ for DS Aqr and $\log \epsilon(N)<6.5$ for V453 Oph. $\log (g f)$ values for the three N I lines were taken from Hibbert et al. (1991).

determined by the effective temperature $\left(T_{\text {eff }}\right)$, gravity $(\log (g))$ and metallicity $([\mathrm{Fe} / \mathrm{H}])$. They are computed with a constant microturbulent velocity $\left(\xi_{\mathrm{t}}=2 \mathrm{~km} \mathrm{~s}^{-1}\right)$ for the opacity distribution functions.

We derived the photospheric model parameters solely using spectroscopic criteria. A temperature is determined by forcing the abundance derived from Fe I lines to be independent of their excitation potential (EP). The large range in $\mathrm{EP}$ and the very large number of lines (V453 Oph: $\mathrm{EP}=0.0-4.4 \mathrm{eV}, N_{\mathrm{FeI}}=$ $104, N_{\mathrm{Fe} I I}=32 ;$ DS Aqr: $\mathrm{EP}=0.0-5.0 \mathrm{eV}, N_{\mathrm{Fe}}=267$, $N_{\mathrm{Fe} \text { II }}=49$ ) ensures an accurate identification of this temperature. This is shown in Fig. 4 and confirmed by other elements with a large number of detected lines (e.g. Ti and $\mathrm{Cr}$ ). 


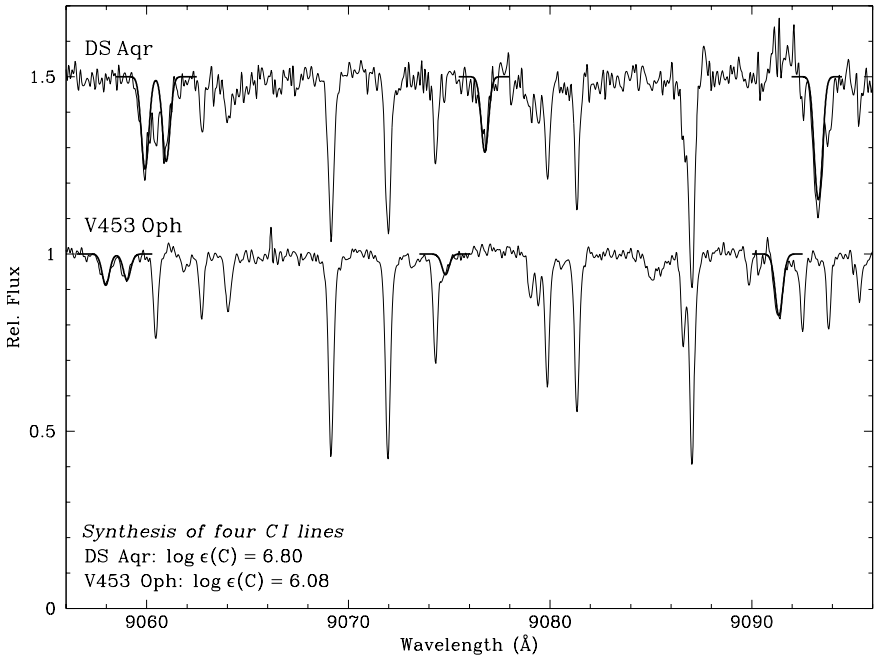

Fig. 6. Synthesis of the four CI lines used in the abundance analysis. These four lines turned out to be the only ones from which a reliable carbon abundance could be derived. The observed spectra are shown with a thin line; the syntheses with a thick line. The spectra are not velocity corrected, providing that the telluric lines are shown at their rest wavelength, and that they can be easily recognised. The macroturbulent velocity used in the syntheses is $8 \mathrm{~km} \mathrm{~s}^{-1}$ for DS Aqr and $10 \mathrm{~km} \mathrm{~s}^{-1}$ for $\mathrm{V} 453 \mathrm{Oph}$. $\log (g f)$ values for these four lines were taken from Hibbert et al. (1993).

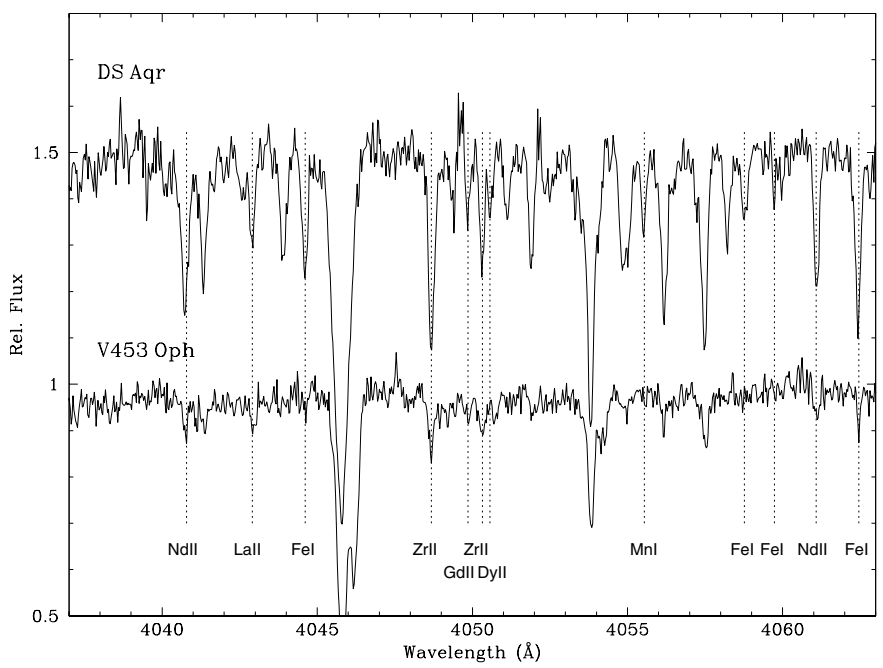

Fig. 7. The velocity corrected spectra of the programme stars showing some of the used s-process lines. For V453 Oph, the s-process lines are almost the only visible lines in the spectrum.

The surface gravity was determined demanding ionisation balance between Fe I and Fe II lines. The microturbulent velocity was estimated requiring the independence of the abundance derived from Fe I lines of the reduced equivalent width. The resulting stellar parameters are shown in Table 4, where the $\mathrm{Fe}$ abundance is determined using an input model with a metallicity of $[\mathrm{M} / \mathrm{H}]=-2.0$ and $[\mathrm{M} / \mathrm{H}]=-1.5$ for $\mathrm{V} 453 \mathrm{Oph}$ and DS Aqr respectively. We used the conservative errors on the model atmosphere parameters of $\Delta T_{\text {eff }}=250 \mathrm{~K}$ and $\Delta \log (g)=$ 0.5 (see Fig. 4). The error on the microturbulent velocity is adopted to be $\Delta \xi_{\mathrm{t}}=0.5 \mathrm{~km} \mathrm{~s}^{-1}$.
Table 4. The stellar parameters of the stars, as determined using only spectroscopic criteria. The reported error on the Fe abundance is only due to line-to-line scatter. See text for more details.

\begin{tabular}{lcccc}
\hline \hline Name & $T_{\text {eff }}(\mathrm{K})$ & $\log (g)$ & $\xi_{\mathrm{t}}\left(\mathrm{km} \mathrm{s}^{-1}\right)$ & {$[\mathrm{Fe} / \mathrm{H}]$} \\
\hline V453 Oph & 6250 & 1.5 & 3.0 & $-2.23 \pm 0.12$ \\
DS Aqr & 5750 & 0.5 & 3.5 & $-1.62 \pm 0.12$ \\
\hline
\end{tabular}

\section{Abundance analysis}

\subsection{Homogeneous analysis}

Severe selection criteria were used to incorporate or discard an absorption line in the analysis. The selected absorption lines have a small equivalent width $(E W<150 \mathrm{~m} \AA)$ and a clear symmetric profile. Blended lines were only taken into account if clearly present and unblended in the other star. The abundances derived from these lines are shown in Table 5.

The abundance of the $\mathrm{CNO}$ elements is always difficult to determine, particularly for such low metallicity stars. For C, the well-known multiplet at $7115 \AA$ is undetectable for both stars. Therefore, a spectrum synthesis was performed using four $\mathrm{C}$ lines around $9070 \AA$ of which the result is shown in Fig. 6. Telluric blending, important in this part of the spectrum, is easily identified by comparing the non-velocity-corrected spectra of both stars, as shown in Fig. 6. A reliable nitrogen abundance is even more difficult to obtain, since the only $\mathrm{N}$ line that is clearly present in our spectrum is weakly blended by Si I and S I lines. As a consequence, we were only able to deduce upper limits for nitrogen, being $\log \epsilon(N)<6.6$ for DS Aqr and $\log \epsilon(N)<6.5$ for V453 Oph. The derivation of these upper limits is shown in the synthesis in Fig.5. The oxygen abundance is primarily based on the strong (non-LTE sensitive) $7774 \AA$ multiplet. For both stars this resulted in a slight underabundance of $\mathrm{C}$ and an overabundance of $\mathrm{N}$ and $\mathrm{O}$, as reported in Table 5. This $\mathrm{N}$ overabundance is probably a consequence of the first dredge-up for both stars, while the $\mathrm{O}$ overabundance is likely of initial composition (e.g. Boesgaard et al. 1999).

Abundances are hard to obtain from sodium (Na) to sulphur (S) due to the lack of lines. However most of the $\alpha$-elements ( $\mathrm{Mg}, \mathrm{Si}, \mathrm{Ca}$ and $\mathrm{Ti}$ ) were detected, showing a mean of $[\alpha / \mathrm{Fe}]=0.3$ for both stars, which is expected for their metallicity (Lambert 1987; Gratton \& Sneden 1991). The Al abundance found for $\mathrm{V} 453 \mathrm{Oph}([\mathrm{Al} / \mathrm{Fe}]=-0.59)$ differs significantly from this, but it is in agreement with the trend of the $\mathrm{Al}$ abundance seen in metal-poor stars (e.g. Gratton \& Sneden 1988). The intermediate mass elements from $\mathrm{V}$ to $\mathrm{Zn}$ are easier to determine. These elements follow the Fe abundance more closely except for $\mathrm{Cr}$ and $\mathrm{Mn}$. While for Mn the underabundance in both objects is expected (e.g. Gratton \& Sneden 1991), the rather large underabundance of $\mathrm{Cr}$ in DS Aqr is not.

A specific procedure was used to find all available lines for the s-process elements (Y, Zr, Ba, La, Ce, $\mathrm{Nd}$ and $\mathrm{Sm}$ ). All lines in the VALD database were used to estimate their equivalent width for the assumed stellar parameters. All lines were then measured in the spectra according to their estimated strength up to the detection limit $(E W \sim 5 \mathrm{~m} \AA)$. Using this method, we maximised the number of lines per species and avoided 
Table 5. Chemical composition of DS Aqr and V453 Oph as derived from the homogeneous abundance analysis. For every ion the abundance is listed absolute and relative to iron. DREAM means that the abundance of Ce II is determined using atomic data obtained from the DREAM database. The reported Eu abundance is corrected for hyperfine splitting. Also listed is the solar abundance $\left(\log \left(\epsilon_{\odot}\right)\right)$, taken from the same references as in Reyniers et al. (2004) except for Dy, where we used the solar abundance of Biémont \& Lowe (1993). Although more recent data exist, the values that were used, ensure that the employed $\log (g f)$ values are consistent with the solar abundances. The line-to-line scatter $\left(\sigma_{\mathrm{ltt}}\right)$ is reported if more than one line was used, together with the number of lines used to determine the abundance $(N)$ and their mean equivalent width $(\bar{W})$. Also reported is the error on the abundances relative to iron $\left(\sigma_{\text {tot }}\right)$, determined as discussed in Sect. 4.2. This table ends with the light and heavy s-process indices (respectively [1s/Fe] and [hs/Fe]) for the homogeneous analysis. The $[1 \mathrm{~s} / \mathrm{Fe}]$ index is the mean of the $\mathrm{Y}$ and $\mathrm{Zr}$ abundance and the $[\mathrm{hs} / \mathrm{Fe}]$ is computed using the mean of the Ba, La and Nd abundance. Clearly V453 Oph shows a significant overabundance for the s-process elements, while DS Aqr does not.

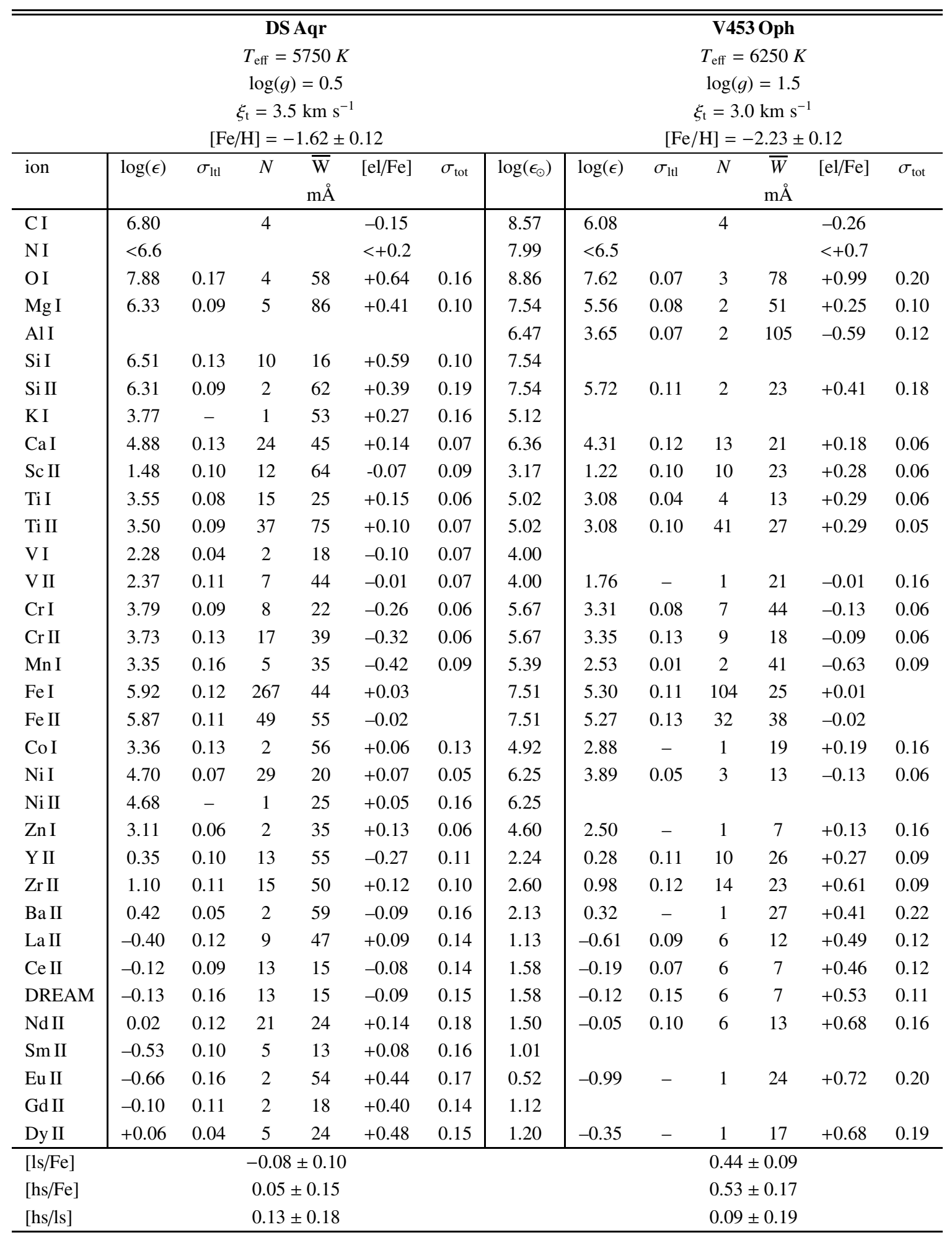


measuring blends. Some of the s-process lines used in the analysis are shown in Fig. 7. Only for Ba was the abundance estimated using fewer than five lines. The abundance of Ce II, obtained using the atomic data from the DREAM database (Palmeri et al. 2000) is also reported. Using this data instead of the VALD data, the abundance of Ce remains fixed for DS Aqr, while it increases for V453 Oph.

Table 5 gives the abundances of the light (ls) and heavy (hs) s-process elements. The light s-elements have a neutron number close to the magic number 50 ( $\mathrm{Y}$ and $\mathrm{Zr}$ ), while the heavy s-elements cluster around the magic neutron number $82(\mathrm{Ba}$, $\mathrm{La}, \mathrm{Ce}, \mathrm{Pr}, \mathrm{Nd}$ and $\mathrm{Sm})$. The indices indicate a mild overabundance of both ls as hs elements for V453 Oph, while for DS Aqr the s-process elements scale with Fe.

The Eu abundance was determined for both objects. However, Eu lines are known to have a strong hyperfine splitting (hfs). A reliable Eu abundance can therefore only be derived when this effect is taken into account. The Eu abundance of DS Aqr is based on two Eu II lines: the resonance line at $4129.72 \AA\left(W_{\lambda}=94 \mathrm{~m} \AA\right)$ and the line at $6645.06 \AA(14 \mathrm{~m} \AA)$; the Eu abundance of V453 Oph is derived solely from the resonance line $(24 \mathrm{~m} \AA$ ). A synthesis of these lines including their hyperfine components showed that the weak lines are only affected by hfs in their profile, and not in their equivalent width. For the resonance line in DS Aqr, however, we found a small but significant effect: the difference in abundance between a hfs and a non-hfs treatment of this line is $-0.06 \mathrm{dex}$. Hyperfine A and B constants for this synthesis were taken from Lawler et al. (2001b) and the solar isotopic composition of Eu was applied. The Eu abundance reported in Table 5 is the hfs-corrected abundance. A comparison between the Eu and Dy abundance gives $[\mathrm{Dy} / \mathrm{Eu}]=0.04$ for both stars. This value is in agreement with Burris et al. (2000) where a [Dy/Eu] abundance very close to zero was found for all metallicities $(-3.0<[\mathrm{Fe} / \mathrm{H}]<-1.0)$.

\subsection{Error analysis}

Considering the absolute abundance determination $(\log (\epsilon))$, the line-to-line scatter $\left(\sigma_{\mathrm{lt}}\right)$ represents the variance of the obtained values. Hence, if more than about five lines were used in the abundance determination, this scatter is representative of the non-systematic errors on the abundances. As indicated in Table 5, the typical line-to-line scatter is $0.1 \mathrm{dex}$.

Systematic and consequently more important errors on the derived abundances are mainly due: uncertainties on the determination of atmospheric parameters, systematic errors in the $\log (g f)$ values and the presence of non-LTE effects. To determine how sensitive the abundances are to the stellar parameters, the abundances were recalculated changing $T_{\text {eff }}, \log (g)$ and $\zeta_{\mathrm{t}}$ with the assessed error (see Sect. 3.3). From this test, it appears that the abundance determination is most sensitive to the effective temperature. The total error due to uncertainties in stellar parameters is given by adding all independent contributions:

$\sigma_{\text {mod }}=\sqrt{\sigma_{T_{\text {eff }}^{2}}^{2}+\sigma_{\log (g)}^{2}+\sigma_{\zeta_{\mathrm{t}}}^{2}}$.

The systematic errors due to the possibly biased $\log (g f)$ values and due to non-LTE effects are more difficult to determine.
Because of the similar effective temperature and gravity of both stars, a large reduction of these errors is guaranteed in a relative abundance analysis (see Sect. 5.3).

In Table 5, the total error on the mean $\left(\sigma_{\text {tot }}\right)$ of the $[\mathrm{el} / \mathrm{Fe}]$ values is shown. This error is determined by adding the errors on the mean due: uncertainties in the model atmosphere $\left(\sigma_{\bmod }\right)$, the line-to-line scatter on the absolute abundances (defaulted to $\sigma_{\mathrm{ltl}}=0.15$ if only one line was used) and the error on the Fe abundance $\left(\sigma_{\mathrm{Fe}}\right)$. The employed formula is:

$\sigma_{\mathrm{tot}}=\sqrt{\left(\frac{\sigma_{\mathrm{ltl}}}{\sqrt{N_{\mathrm{el}}}}\right)^{2}+\left(\sigma_{\mathrm{mod}}\right)^{2}+\left(\frac{\sigma_{\mathrm{Fe}}}{\sqrt{N_{\mathrm{Fe}}}}\right)^{2}}$

\section{Discussion of chemical analysis}

\subsection{Comparison with the literature}

The chemical patterns of both stars were previously analysed in the literature. For DS Aqr, two analyses were performed (Giridhar et al. 1998, 2000), with the latter one being more elaborate, while for V453 Oph only one analysis was performed (Giridhar et al. 1998).

The analysis in the literature for V453 Oph and the one presented here resulted in the same metallicity and very similar $[\mathrm{el} / \mathrm{Fe}]$ values. Only for $[\mathrm{C} / \mathrm{Fe}]$ and $[\mathrm{O} / \mathrm{Fe}]$, being respectively 0.5 dex lower and 0.8 dex higher in this analysis, there are discrepancies. Neither previous abundances are compatible with our spectra. For the other elements reported in both analyses, the mean difference relative to $\mathrm{Fe}$ is only 0.12 dex. The high radial velocity, indicating membership of pop. II stars, strengthens the very low metallicity of the star V453 Oph.

The metallicity reported in Giridhar et al. (2000) for DS Aqr, $[\mathrm{Fe} / \mathrm{H}]=-1.1$, differs significantly from the one reported here. However our analysis uses a much larger number of lines of Fe I and Fe II, so we are confident in the established value. For the fractional abundances, however, the [el/Fe] values in Giridhar et al. (2000) differ on average by only $0.06 \mathrm{dex}$ from those reported here. Since we focused on s-process lines, we use many more available lines to derive quantitative data.

We conclude that our spectra are clearly not compatible with some absolute abundance values found in the literature. Our $S / N$ is higher and our abundance analysis covers many more elements, in particular more lines for s-process elements.

\subsection{Depletion process}

As expected for RVC variables, the depletion process was not efficient in the investigated objects. This is clearly demonstrated in Fig. 8, where the abundances are plotted versus the condensation temperature (Lodders \& Fegley 1988). A star subjected to the depletion process shows a lowered abundance of elements with a higher condensation temperature. This is clearly not observed in either variable. Therefore, the derived abundances represent the initial composition of the stars, possibly altered by their internal evolution and/or accretion of enriched material from a companion. 

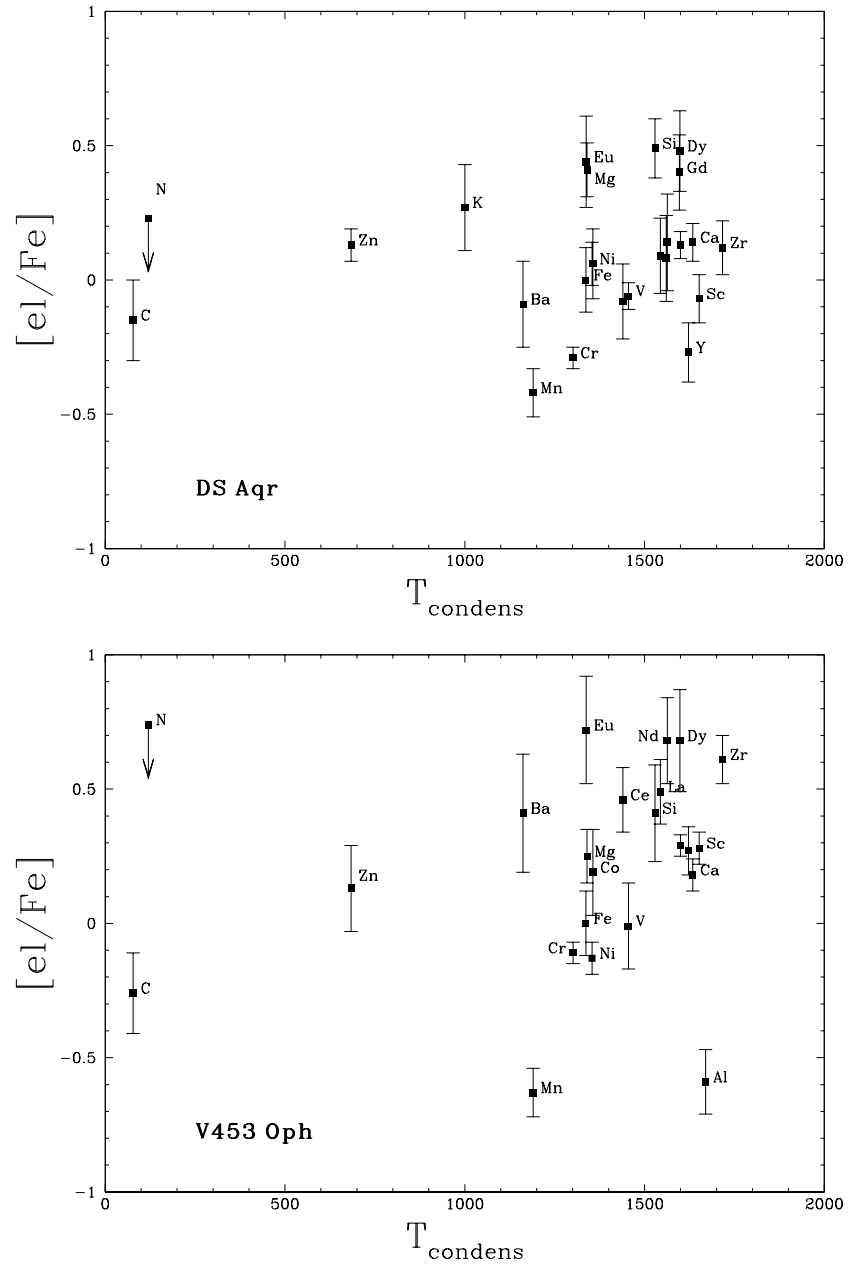

Fig. 8. The derived abundances versus the condensation temperature for DS Aqr (top) and V453 Oph (bottom). These figures indicate that no depletion process occurred.

\section{3. s-process overabundances}

The s-process indices reported in Table 5 in combination with the abundances of the individual s-process elements indicate that DS Aqr shows the expected abundances for a photosphere unaltered by internal evolution (e.g. François et al. 2003, and references therein), while for V453 Oph the overabundance of heavy elements indicates mixing of nucleosynthetic elements in the photosphere.

To constrain possible systematic uncertainties in the reported abundances, a strictly relative analysis was performed, which uses only spectral lines detected in both objects. The results of this analysis are listed in Table 6. By comparing V453 Oph with the results of DS Aqr, we assume that the latter is a good tracer of the mean Galactic trend. The individual relative abundances given in Table $6(\Delta[\mathrm{el} / \mathrm{Fe}])$ are thus to be interpreted as the abundance characteristics of V453 Oph with respect to unevolved objects at similar metallicity that follow the mean Galactic trend. This analysis provides a solid basis for the proposition that V453 Oph is a metal deficient pop II RV Tauri star with a significant enrichment in heavy elements.

The errors on the mean of the relative abundances $\left(\sigma_{\text {tot }}\right)$ were estimated assuming that uncertainties due to the model
Table 6. Results from the relative analysis of DS Aqr and V453 Oph only using the lines that were observed in both spectra. In the column labelled $\Delta[\mathrm{el} / \mathrm{Fe}]$, the difference in abundance relative to $\mathrm{Fe}$ for V453 Oph minus the one for DS Aqr is given. The other notations are similar as in Table 5. This analysis shows clearly an overabundance of s-process elements in V453 Oph because possible bias effects due to uncertain $\log (g f)$ values are eliminated in a relative study.

\begin{tabular}{|c|c|c|c|c|}
\hline \multirow[b]{2}{*}{ ion } & \multicolumn{4}{|c|}{$\begin{array}{l}\text { Relative } \\
\text { analysis }\end{array}$} \\
\hline & $\sigma_{\mathrm{ltl}}$ & $\Delta[\mathrm{el} / \mathrm{Fe}]$ & $N$ & $\sigma_{\text {tot }}$ \\
\hline $\mathrm{Mg} \mathrm{I}$ & 0.04 & -0.13 & 2 & 0.03 \\
\hline Si II & 0.02 & +0.02 & 2 & 0.02 \\
\hline $\mathrm{Ca} \mathrm{I}$ & 0.10 & +0.11 & 12 & 0.03 \\
\hline Sc II & 0.10 & +0.33 & 7 & 0.04 \\
\hline Ti I & 0.01 & +0.20 & 2 & 0.01 \\
\hline Ti II & 0.07 & +0.18 & 24 & 0.02 \\
\hline V II & - & -0.02 & 1 & 0.15 \\
\hline $\mathrm{CrI}$ & - & +0.25 & 1 & 0.15 \\
\hline Cr II & 0.06 & +0.25 & 8 & 0.02 \\
\hline Co I & - & +0.24 & 1 & 0.15 \\
\hline Ni I & 0.04 & -0.16 & 2 & 0.03 \\
\hline $\mathrm{Zn} \mathrm{I}$ & - & +0.04 & 1 & 0.15 \\
\hline Y II & 0.12 & +0.53 & 8 & 0.04 \\
\hline $\mathrm{Zr}$ II & 0.09 & +0.47 & 11 & 0.03 \\
\hline $\mathrm{Ba}$ II & - & +0.54 & 1 & 0.15 \\
\hline La II & 0.10 & +0.41 & 4 & 0.05 \\
\hline Ce II & 0.02 & +0.52 & 2 & 0.02 \\
\hline DREAM & 0.02 & +0.52 & 2 & 0.02 \\
\hline Nd II & 0.16 & +0.55 & 5 & 0.07 \\
\hline $\mathrm{Eu}$ II & - & +0.39 & 1 & 0.15 \\
\hline$[\mathrm{ls} / \mathrm{Fe}]$ & \multicolumn{4}{|c|}{$0.50 \pm 0.04$} \\
\hline [hs/Fe] & \multicolumn{4}{|c|}{$0.50 \pm 0.10$} \\
\hline$[\mathrm{hs} / \mathrm{ls}]$ & \multicolumn{4}{|c|}{$0.00 \pm 0.11$} \\
\hline
\end{tabular}

atmospheres cancel out because both stars have similar atmospheric parameters. Thus we used a formula similar to Eq. (2), excluding the uncertainty on the model atmospheres, but including the uncertainty on the Fe abundance of both stars. The resulting errors on the relative abundances are shown in Table 6.

\subsection{Comparison with $\mathrm{CH}$-giants}

We performed a comparison of the V453 Oph s-enrichment with those reported for $\mathrm{CH}$ stars of similar metallicity. $\mathrm{CH}$-stars are cool C-rich objects of low metallicity showing enhanced s-process lines. A distinction is made between early-type and late-type $\mathrm{CH}$-stars: early-type $\mathrm{CH}$-stars are probably binaries which are extrinsically enriched (e.g. McClure \& Woodsworth 1990), while late-type $\mathrm{CH}$-stars combine bright absolute magnitudes and high ${ }^{12} \mathrm{C} /{ }^{13} \mathrm{C}$ ratios and are therefore believed to be intrinsically enriched (Van Eck et al. 2003).

Standard s-process scenarios predict the production of mainly $\mathrm{Pb}$ for the low-metallicity $\mathrm{CH}$-stars $(\mathrm{Pb} / \mathrm{Ba}$ ratios of 70 


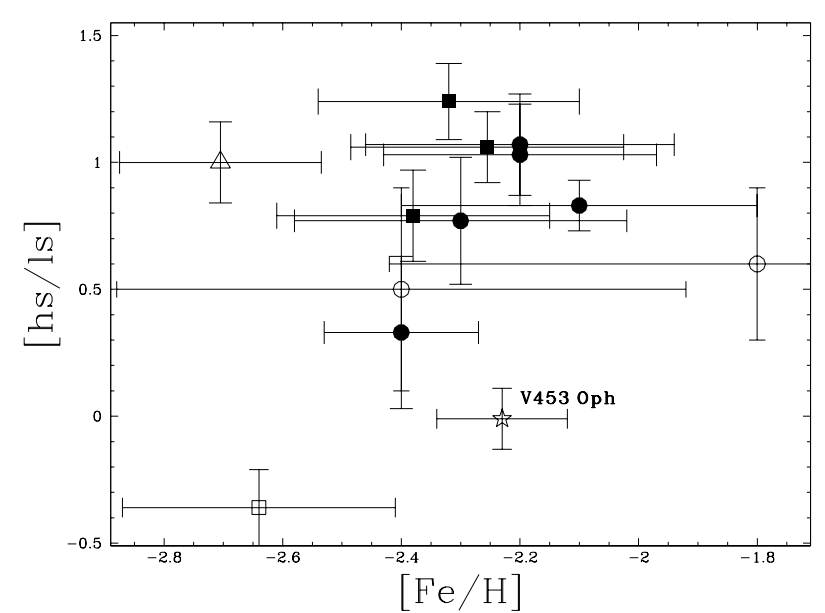

Fig. 9. In a narrow metallicity interval, the literature [hs/ls] values and those for V453 Oph are plotted. We refer for the definition of the ls and hs indexes to the text. The $\mathrm{CH}$ stars with the expected $\mathrm{Pb}$ overabundance are depicted with filled symbols, while the others are represented using open symbols. The circles are taken from Van Eck et al. (2003), the upper triangles from Aoki et al. (2001) and the squares from Aoki et al. (2002). The star V453 Oph is shown as an asterisk and labelled in the graph. For this star, no $\mathrm{Pb}$ abundance was determined.

are predicted for $[\mathrm{Fe} / \mathrm{H}]=-1.3$ by Goriely \& Mowlavi 2000). Some of the studied $\mathrm{CH}$-stars show this extreme $\mathrm{Pb}$ enrichment, while others do not (e.g. Van Eck et al. 2003, and references therein). A comparison of the reported s-process enrichments is shown in Fig. 9. In this figure, the [hs/ls] value is plotted as a function of metallicity. The ls value is taken as the abundance of $\mathrm{Zr}$ and the hs value as the mean of the abundances of La, Ce and Nd. Only stars with all these abundances determined were taken into account. In Fig. 9 a large spread in [hs/ls] is observed for very similar metallicities. Although V453 Oph is situated in a less dense region of this plot, some $\mathrm{CH}$-giants have similar [hs/ls] indices. Extremely Pb-enriched as well as not $\mathrm{Pb}$-enriched objects are found with a $[\mathrm{hs} / \mathrm{ls}]$ value similar to $\mathrm{V} 453 \mathrm{Oph}$. Note that the determination of the $\mathrm{Pb}$ abundance in the hot V453 Oph star is difficult and only an upper limit of $[\mathrm{Pb} / \mathrm{Fe}]<2$ could be determined.

\subsection{Other RV Tauri stars with s-process enrichment}

To our knowledge, up to now only one other RV Tauri object with a clear s-process enrichment has been detected: V1 in $\omega$ Cen (Gonzalez \& Wallerstein 1994). This RV Tau star with a spectroscopic class on the B-C boundary (Wallerstein \& Cox 1984) also shows mild s-process overabundances $([\mathrm{ls} / \mathrm{Fe}]=0.6$ and $[\mathrm{hs} / \mathrm{Fe}]=0.5)$. It is unclear whether this enrichment is intrinsic, or if it is due to the well-studied but poorly understood self-enrichment of $\omega$ Cen (e.g. Tsujimoto \& Shigeyama 2003, and references therein). The fact that the less evolved variables V48 and V29, also studied in Gonzalez \& Wallerstein (1994), display the same s-process overabundances as V1, is in favour of the self-enrichment scenario. V1, however, also shows a small, but significant, overabundance in carbon compared to these two other variables $(\Delta[\mathrm{C} / \mathrm{Fe}]=0.9$ and 0.5 for $\mathrm{V} 48$ and $\mathrm{V} 29$ respectively). Thus it appears that it is not only possible to enhance solely the s-process elements (V453 Oph) in RV Tauri variables, it is also possible to enhance only the $\mathrm{C}$ abundance ( $\omega$ Cen V1) without strongly enriching the star with s-process elements.

\section{V453 Oph, initial, extrinsic or intrinsic heavy element overabundances?}

The most important abundance characteristic of V453 Oph is that it is a genuine low-metallicity star with $[\mathrm{Fe} / \mathrm{H}]=-2.2$ showing a significant heavy element overabundance, but without any $\mathrm{C}$-enrichment. Moreover, the $\mathrm{N}$-abundance is too low to explain the $\mathrm{C}$ deficiency by $\mathrm{CN}$ cycling. The luminosity and atmospheric parameters of $\mathrm{V} 453 \mathrm{Oph}$ are compatible with an object of low initial mass (typically $M \simeq 0.8 M_{\odot}$ ) in its postAGB phase of evolution.

We explored several possible scenarios to explain the observed abundances: special chemical characteristics of the parental cloud; enrichment by a binary companion and intrinsic s-process enrichment by dredge-up.

\subsection{Parental cloud anomalies}

At low metallicity, the Galaxy was not well mixed so there is a large star-to-star scatter in the initial abundance ratios of unevolved stars of low iron abundance $([\mathrm{Fe} / \mathrm{H}] \leq-2)$. The chemical composition of low-metallicity stars may reflect strong initial heavy element enrichment of the parental cloud. To test whether this may have been the case for V453 Oph, we scanned the recent literature for good quality data of individual nonevolved stars. We compared both the overabundances of individual neutron capture elements as well as the observed distribution, since the latter is a good tracer of the nucleosynthesis production site.

Massive stars are believed to be major contributors of neutron capture elements in the Galaxy, with a most probable enrichment in r-process nuclei produced during the supernova explosion, but also of the so-called weak s-process component responsible for the production of s-nuclei up to $A \simeq 90$ during core He-burning. Low-metallicity stars polluted by supernovaenriched material are therefore likely to show, in addition to an s-process enrichment in elements up to $\mathrm{Zr}$, a strong r-process signature. In recent years, it became clear that this picture is oversimplified and recent observations of a large sample of low-metallicity stars show evidence of the main and strong components of the s-process in individual unevolved stars with metallicities as low as $[\mathrm{Fe} / \mathrm{H}]=-3$ (Simmerer et al. 2004). These authors focus on the study of $\mathrm{La}$ and $\mathrm{Eu}$ abundances of about 160 giant and dwarf stars in the metallicity range $-3<[\mathrm{Fe} / \mathrm{H}]<+0.3$. In Fig. 10 we compare the abundances of V453 Oph with the sample of Simmerer et al. (2004). The La enrichment is above the level observed in objects of similar metallicity, but the excess is mild.

Detailed chemical studies of very low-metallicity unevolved stars tend to focus on the few objects which are known to show this clear dominant r-process signature. The most famous example in this respect is the ultra-metal-deficient star CS 22892-052 (Sneden et al. 2003, and references therein). 


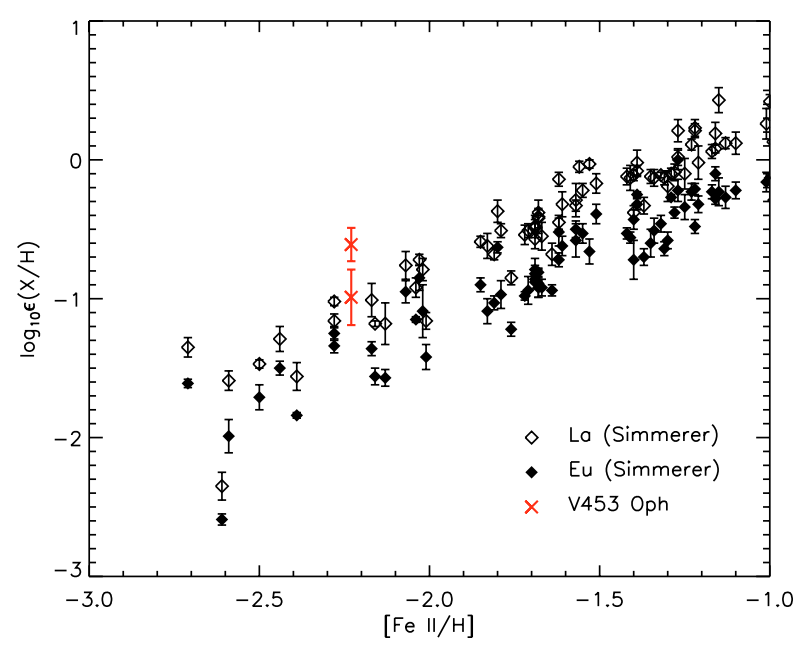

Fig. 10. A comparison of the $\mathrm{La}$ and Eu abundance of V453 Oph with the values found for non-enriched objects in Simmerer et al. (2004). Although the excess is small, the La abundance of V453 Oph is above the values observed for non-enriched objects.

We compared the heavy element distribution of V453 Oph with the well studied r-process enriched halo giant BD+17.3248 (Cowan et al. 2002). Both stars have the same metallicity and show similar overabundances of some individual heavy elements. The difference between both distributions is most apparent in Eu and Dy. When both distributions are normalised to $\mathrm{Zr}$, both the Eu and Dy abundances of BD+17.3248 are 0.6 dex larger than what is observed in V453 Oph.

In Table 7 we compare the V453 Oph overabundances to those found in non-evolved objects of similar metallicities from the extensive study of Burris et al. (2000). At metallicity $[\mathrm{Fe} / \mathrm{H}]=-2.2$, the $\mathrm{Y}$ abundance of $\mathrm{V} 453 \mathrm{Oph}([\mathrm{Y} / \mathrm{Fe}]=+0.3$ and $[\mathrm{Y} / \mathrm{Fe}]=+0.5$ in DS Aqr) is clearly above the mean value of -0.2 from Burris et al. (2000) sample. Outliers to this mean Galactic trend exist, however, but the highest observed value in this sample of 10 stars is about $[\mathrm{Y} / \mathrm{Fe}]=+0.0$ in the metallicity range of $\mathrm{V} 453 \mathrm{Oph}$. The scatter in the $[\mathrm{Zr} / \mathrm{Fe}]$ ratio at metallicity around -2.2 is similar, but here again V453 Oph is significantly more $\mathrm{Zr}$-rich than the other objects. The La overabundance with respect to the Galactic mean is confirmed in this comparison. For the heavy elements $\mathrm{Nd}, \mathrm{Eu}$ and Dy, the general picture is not as clear. In V453 Oph, they tend to be as abundant as in the Burris et al. (2000) sample, although some stars do exhibit similar enrichments.

In conclusion, although the $[\mathrm{Eu} / \mathrm{Fe}]$ and $[\mathrm{Dy} / \mathrm{Eu}]$ abundances in both V453 Oph and DS Aqr indicate an initial enrichment of r-process nature, the relatively large $\mathrm{Y}, \mathrm{Zr}, \mathrm{Ba}$ and $\mathrm{La}$ overabundances of V453 Oph with respect to DS Aqr and the low-metallicity stars in the Burris et al. (2000) sample clearly points to an additional s-process enrichment in V453 Oph. If the actual chemical distribution of $\mathrm{V} 453 \mathrm{Oph}$ reflects the initial composition of the parental cloud, this cloud must have been subject to some s-process enrichment capable of producing elements up to the Ba peak. However, up to now, only AGB stars are believed to be responsible for this corresponding main component of the s-process, massive stars being capable of producing elements up to $\mathrm{Zr}$ only.
Table 7. Comparison between the enrichments found in V453 Oph and the abundances found in unevolved low-metallicity stars compiled by Burris et al. (2000). The mean and standard deviation of the values obtained for the 18 stars in the metallicity range $-2.4<[\mathrm{Fe} / \mathrm{H}]<$ -2.0 is given. Not all data are available for all stars, so the number of stars used in the compilation is given in the last column.

\begin{tabular}{lcccc}
\hline \hline & V453 Oph & Mean & $1 \sigma$ & $n$ \\
\hline$[\mathrm{Y} / \mathrm{Fe}]$ & +0.27 & -0.23 & 0.18 & 10 \\
{$[\mathrm{Zr} / \mathrm{Fe}]$} & +0.61 & +0.17 & 0.25 & 10 \\
{$[\mathrm{Ba} / \mathrm{Fe}]$} & +0.41 & +0.12 & 0.50 & 18 \\
{$[\mathrm{La} / \mathrm{Fe}]$} & +0.49 & +0.05 & 0.28 & 10 \\
{$[\mathrm{Nd} / \mathrm{Fe}]$} & +0.68 & +0.14 & 0.38 & 10 \\
{$[\mathrm{Eu} / \mathrm{Fe}]$} & +0.72 & +0.27 & 0.38 & 12 \\
{$[\mathrm{Dy} / \mathrm{Eu}]$} & +0.68 & +0.37 & 0.35 & 10 \\
\hline
\end{tabular}

\subsection{Extrinsic enrichment}

The enrichment we see in V453 Oph may come from the pollution of a putative companion similar to the extrinsically enriched $\mathrm{CH}$ stars discussed in Sect. 5.4. Binarity among dusty RV Tauri stars may be widespread (Van Winckel et al. 1999b; De Ruyter et al. 2005) and the arguments are either direct (with direct radial velocity measurements), or indirect (SED characteristics pointing to the presence of a dusty disc instead of an outflow). Note that only the dusty RV Tauri stars are thought to be binaries in which the companion is probably not a white dwarf but an unevolved main sequence star. No binary RV Tauri star is definitely identified with a White Dwarf companion.

Unfortunately, we do not have detailed radial velocity monitoring data for V453 Oph. In V453 Oph, no dust-excess is observed so the above mentioned indirect evidence for binarity is lacking. Also, the intrinsic luminosity determined by the $\mathrm{P}-\mathrm{L}$ relation is large, pointing to a genuine post-AGB nature. Note that extrinsically enriched objects are rare : Giant barium stars (extrinsically enriched Giants with white dwarf companions) represent only $1 \%$ of the giant G-K stars (e.g. Jorissen 2004).

We conclude that, although we cannot exclude the possibility that V453 Oph belongs to a binary system which has been polluted by an AGB star that is now a White Dwarf companion, this hypothesis is not very likely. Moreover, the $\mathrm{C}$ deficiency associated with the s-process enrichment remains difficult to explain by standard nucleosynthesis models (see Sect. 7). This scenario presents however the advantage that the White Dwarf progenitor responsible for the s-process nucleosynthesis can a priori be of any initial mass (below some $8 M_{\odot}$ ).

\subsection{Intrinsic enrichment}

The most natural scenario for a post-AGB star to become overabundant in s-process elements is that the object was subject to internal nucleosynthesis followed by dredge-up episodes during its previous AGB phase. The most favoured s-process model is associated with the partial mixing of protons (PMP) into the radiative C-rich layers at the time of the 3DUP (Gallino et al. 1998; Goriely \& Mowlavi 2000). There is, however, no general agreement on what physical mechanism can be 
responsible for the injection of protons into the ${ }^{12} \mathrm{C}$-rich layers. Furthermore, in this scenario, the s-process surface enrichment is bound to be associated with a stronger $\mathrm{C}$ pollution.

With a metallicity of -2.2 and a luminosity $L=2400 \pm$ $1600 L_{\odot}$, this post-AGB star must have evolved from a lowmass main sequence star (typically $M \simeq 0.8 M_{\odot}$ ). For such stars, it remains unknown if any PMP may take place in the C-rich region, and even if any third dredge-up can intrinsically enrich the stellar surface. The results of our abundance analysis are consequently difficult to reconcile with this evolutionary path as well.

\section{S-process models and observations}

As explained in the previous section, none of the standard scenarios can a priori explain the present observations. It is clear that the abundances of V453 Oph challenge our current understanding of the s-process. On the one hand, s-process nucleosynthesis during core He-burning in massive stars can not account for a significant production of s-elements up to the $\mathrm{Ba}$ peak. On the other hand, any AGB s-process models within the PMP scenario would lead to a concomitant and significant production of $\mathrm{C}$ which is not observed. More specifically, as described in detail in (Deroo et al. 2005), the PMP model in the intrinsic enrichment scenario could in principle lead to an abundance distribution close to the observed one, but would simultaneously pollute the stellar envelope with a $\mathrm{C}$ overabundance of the order of $[\mathrm{C} / \mathrm{Fe}]=1.5$. The major specificity of this $\mathrm{PMP}$ s-process is the prediction of a large $\mathrm{Pb}$ overabundance that cannot be confirmed by the present observations (the upper limit established from the observations is $[\mathrm{Pb} / \mathrm{Fe}] \leq 2.0$ ).

As shown by Deroo et al. (2005), there is however one s-process model that could explain to some extent at least part of the observations. This is the so-called convective s-process model that can possibly develop at the base of hot thermal pulses in massive AGB stars. Assuming again the extrinsic enrichment scenario to prevail, we can imagine that a massive AGB companion star could have polluted V453 Oph by stellar winds. In massive AGB stars, the temperature at the base of the thermal pulse is hot enough for the ${ }^{22} \mathrm{Ne}(\alpha, \mathrm{n})^{25} \mathrm{Mg}$ to become active and be responsible for some s-process nucleosynthesis. The major advantage of this scenario in comparison with the PMP model is that the s-process enrichment in the pulse can reach values similar to or even greater than the $\mathrm{C}$ one. This is due to the large mass extent on which s-process seed nuclei are irradiated by neutrons $\left(M_{\text {pulse }} \sim 10^{-3} M_{\odot}\right)$ compared with the $10^{-5} M_{\odot}$ resulting from the PMP models. The resulting surface overabundances are shown in Fig. 11. Such a dilution gives rise to the corresponding overabundances $[\mathrm{C} / \mathrm{Fe}]=0.1,[\mathrm{~N} / \mathrm{Fe}]=0.6$ and $[\mathrm{Mg} / \mathrm{Fe}]=0.4$, and no $\mathrm{Pb}$ overproduction contrary to the PMP nucleosynthesis. There is however a drawback to this scenario: to reduce the $\mathrm{C}$ pollution, the star should not have endured the third dredge-up episodes. It remains unclear what star may or may not be subject to the third dredge-up, different convective models with or without extra mixing mechanisms leading to significantly different predictions. The presence of a binary companion is an additional source of complexity.

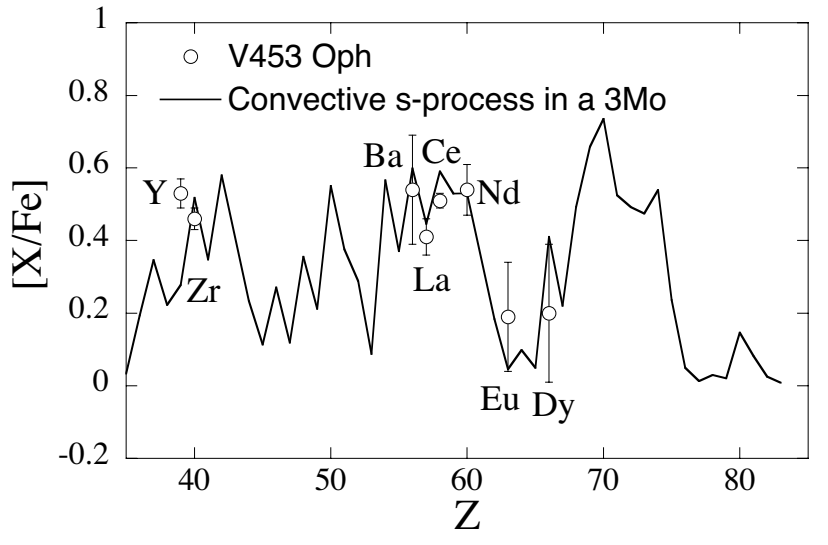

Fig. 11. Comparison of the observed surface abundances (enrichment of V453 Oph with respect to DS Aqr) with the s-process abundances obtained within the convective s-process model in a $3 M_{\odot}$ $Z=0.0001$ model star.

Only in this very specific model and if no 3DUP occurs to enrich the envelope in carbon, the surface enrichment of selements can be reconciled with a low C-content. It is clear that, although the comparison of the calculated and observed abundances is quite satisfactory, the $3 M_{\odot} Z=0.0001$ model star can only have been the putative companion to V453 Oph. Such a massive object of low metallicity must have evolved into a white dwarf by now.

\section{Conclusion}

In this paper, an abundance analysis is presented of two very similar RV Tauri stars of class C, V453 Oph and DS Aqr. Both objects are genuine post-AGB stars, although both have no detected IR excess due to circumstellar dust. These stars differ significantly when we look at their photospheric abundance pattern. While V453 Oph shows an excess of light and heavy s-process elements, DS Aqr shows neither. This conclusion is clearly demonstrated by the relative analysis that was performed between both objects.

The abundances derived for V453 Oph are of particular nucleosynthesis interest, since for the first time, the observed overabundance of a s-process signature is not accompanied by a simultaneous $\mathrm{C}$-enrichment. Moreover, the $\mathrm{N}$ abundance upper limit excludes possible $\mathrm{CN}$ cycle destruction of the dredged-up carbon. The most favoured AGB s-process model based on the partial mixing of protons at the time of the third dredge-up is inevitably accompanied by a larger surface enrichment in $\mathrm{C}$ and therefore fails to account for the present observation.

We explored three evolution scenarios to explain the abundance distribution of V453 Oph. All have specific shortcomings. The first possibility is that the parental cloud was initially polluted by heavy elements. The abundances observed in nonevolved objects of the same metallicity $([\mathrm{Fe} / \mathrm{H}] \sim-2.2)$ show quite some spread, illustrating that the Galaxy was not well mixed at these epochs. Although some of these objects do exhibit significant heavy element overabundances, a comparison with the abundances observed in V453 Oph indicate that its 
parental cloud must have been quite extremely polluted in $\mathrm{r}$ - but also s-elements.

Alternatively, the star may have been enriched during its evolution either by mass transfer or internally. If the star is indeed intrinsically enriched, this would suggest that low-mass objects of low metallicity can be subject to s-process nucleosynthesis without simultaneously polluting their envelope with C. At the present time, it is impossible to reconcile such conditions with our present understanding of AGB stars. If the star belongs to a binary system, the companion may have polluted the actual post-AGB star but here again the same $\mathrm{C}$ deficiency is difficult to reconcile with the traditional s-process scenarios. Moreover, there is still no evidence of a WD companion. Nevertheless, we explored the possibility for a $3 M_{\odot}$ $Z=0.0001$ model star to produce s-elements within the thermal pulses only and conclude that such a convective s-process model could to some extent reconcile the observed s-process enrichment without significant C-enrichment. Clearly, more indepth theoretical and observational studies are needed to further understand if such a scenario could indeed be responsible for the extraordinary chemical composition of the V453 Oph surface.

\section{References}

Alcock, C., Allsman, R. A., Alves, D. R., et al. 1998, AJ, 115, 1921

Aoki, W., Ryan, S. G., Norris, J. E., et al. 2001, ApJ, 561, 346

Aoki, W., Ryan, S. G., Norris, J. E., et al. 2002, ApJ, 580, 1149

Biémont, E., \& Lowe, R. M. 1993, A\&A, 273, 665

Bloecker, T. 1995, A\&A, 299, 755

Boesgaard, A. M., King, J. R., Deliyannis, C. P., \& Vogt, S. S. 1999, AJ, 117, 492

Burris, D. L., Pilachowski, C. A., Armandroff, T. E., et al. 2000, ApJ, 544, 302

Castelli, F., \& Kurucz, R. L. 2004 [arXiv: astro-ph/0405087]

Cowan, J. J., Sneden, C., Burles, S., et al. 2002, ApJ, 572, 861

De Ruyter, S., Van Winckel, H., Dominik, C., Waters, L. H. D., \& Dejonghe, H. 2005, A\&A, 435, 161

Deroo, P., Goriely, S., Siess, L., Reyniers, M., \& Van Winckel, H. 2005, Nucl. Phys. A, in press

Evans, T. L. 1985, MNRAS, 217, 493

François, P., Depagne, E., Hill, V., et al. 2003, A\&A, 403, 1105

Gallino, R., Arlandini, C., Busso, M., et al. 1998, ApJ, 497, 388

Gehrz, R. D. 1972, ApJ, 178, 715

Giridhar, S., Lambert, D., \& Gonzalez, G. 2000, ApJ, 531, 521

Giridhar, S., Lambert, D. L., \& Gonzalez, G. 1998, ApJ, 509, 366

Goldsmith, M. J., Evans, A., Albinson, J. S., \& Bode, M. F. 1987, MNRAS, 227, 143

Gonzalez, G., \& Wallerstein, G. 1994, AJ, 108, 1325

Goriely, S., \& Mowlavi, N. 2000, A\&A, 362, 599
Gratton, R. G., \& Sneden, C. 1988, A\&A, 204, 193

Gratton, R. G., \& Sneden, C. 1991, A\&A, 241, 501

Hibbert, A., Biémont, E., Godefroid, M., \& Vaeck, N. 1991, A\&AS, 88,505

Hibbert, A., Biémont, E., Godefroid, M., \& Vaeck, N. 1993, A\&AS, 99, 179

Jorissen, A. 2004, in Asymptotic Giant Branch Stars, 461

Josafatsson, K., \& Snow, T. P. 1987, ApJ, 319, 436

Jura, M. 1986, ApJ, 309, 732

Kholopov, P. N., Samus, N. N., Frolov, M. S., et al. 1998, in Combined General Catalogue of Variable Stars, 4.1 ed. (II/214A), 0

Krełowski, J., Ehrenfreund, P., Foing, B. H., et al. 1999, A\&A, 347, 235

Kupka, F., Piskunov, N., Ryabchikova, T. A., Stempels, H. C., \& Weiss, W. W. 1999, A\&AS, 138, 119

Lambert, D. L. 1987, A\&A, 8, 103

Lawler, J. E., Bonvallet, G., \& Sneden, C. 2001a, ApJ, 556, 452

Lawler, J. E., Wickliffe, M. E., den Hartog, E. A., \& Sneden, C. 2001b, ApJ, 563, 1075

Lodders, K., \& Fegley, B. 1988, The Planetary Scientist's Companion (Oxford University Press)

Maas, T. 2003, Ph.D. Thesis, University of Leuven

Maas, T., Van Winckel, H., \& Lloyd Evans, T. 2005, A\&A, 429, 297

Mathis, J. S., \& Lamers, H. J. G. L. M. 1992, A\&A, 259, L39

McClure, R. D., \& Woodsworth, A. W. 1990, ApJ, 352, 709

Palmeri, P., Quinet, P., Wyart, J. F., \& Biémont, E. 2000, Phys. Scr., 61,323

Preston, G. W., Krzeminski, W., Smak, J., \& Williams, J. A. 1963, ApJ, 137, 401

Queloz, D., Casse, M., \& Mayor, M. 1999, in IAU Colloq., 170, Precise Stellar Radial Velocities, ASP Conf. Ser., 185, 13

Reyniers, M., Van Winckel, H., Gallino, R., \& Straniero, O. 2004, A\&A, 417, 269

Simmerer, J., Sneden, C., Cowan, J. J., et al. 2004, ApJ, 617, 1091

Sneden, C., Cowan, J. J., Lawler, J. E., et al. 2003, ApJ, 591, 936

Thévenin, F. 1989, A\&AS, 77, 137

Tsujimoto, T., \& Shigeyama, T. 2003, ApJ, 590, 803

Van Eck, S., Goriely, S., Jorissen, A., \& Plez, B. 2003, A\&A, 404, 291

Van Winckel, H. 2003, in Planetary Nebulae: Their evolution and role in the Universe, IAU Symp., 209, 000

Van Winckel, H., \& Reyniers, M. 2000, A\&A, 354, 135

Van Winckel, H., Waelkens, C., \& Waters, L. B. F. M. 1995, A\&A, 293, L25

Van Winckel, H., Waelkens, C., Fernie, J. D., \& Waters, L. B. F. M. 1999a, A\&A, 343, 202

Van Winckel, H., Waelkens, C., Fernie, J. D., \& Waters, L. B. F. M. 1999b, A\&A, 343, 202

Wallerstein, G. 2002, PASP, 114, 689

Wallerstein, G., \& Cox, A. N. 1984, PASP, 96, 677

Waters, L. B. F. M., Trams, N. R., \& Waelkens, C. 1992, A\&A, 262, L37 